\title{
Cyclic Changes of Nerve Fibers in Human Endometrium
}

\author{
Tatsuo Tomita ${ }^{1,2,3^{*}}$, Kuni Mah ${ }^{3}$ \\ ${ }^{1}$ Department of Integrative Bioscience, Oregon Health and Science University, Portland, OR, USA \\ ${ }^{2}$ Department of Pathology, Oregon Health and Science University, Portland, OR, USA \\ ${ }^{3}$ Oregon National Primate Center, Beaverton, OR, USA \\ Email: *tomitat@ohsu.edu
}

Received 1 March 2014; revised 1 April 2014; accepted 8 April 2014

Copyright @ 2014 by authors and Scientific Research Publishing Inc.

This work is licensed under the Creative Commons Attribution International License (CC BY).

http://creativecommons.org/licenses/by/4.0/

(c) (i) Open Access

\begin{abstract}
Objective: The presence of nerve fibers in human endometrium remains unsettled but recent immunocytochemical studies have shown that there was increased innervation in the endometrium from women with endometriosis and some nerve fibers in the normally cycling human endometrium. In the current study, we used uterine tissue cryosections from normal cycling women, which previously provided better immunocytochemical staining for lymphatic vessels than in paraffin sections. Materials and Methods: A total of 16 cases from normally cycling women were included representing menstrual, early proliferative, early to late secretary phase. Neurofilament and CD 56 were used as immunocytochemical markers for nerve fibers with cryosections. Results: There were consistent presence of nerve fibers in myometrium and basalis. Few small nerve fibers were identified in early proliferative endometrium and more nerve fibers were present in lower-half functionalis from mid-secretary phase. Late-secretary functionalis showed less nerve fibers in the upper-half than the lower-half functionalis, implying growing nerve fibers from lower functionalis to upper functionalis in late-secretary phase. Conclusion: Nerve fibers appeared to cyclically grow from basalis to lower functionalis and then from lower functionalis to upper functionalis concomitantly with blood vessels in normally cycling human endometrium. These cycling endometrial nerve fibers consisted mostly of nonmyelinated small nerve fibers, which may transmit pelvic pain in the normally cycling women.
\end{abstract}

\section{Keywords}

Basalis, CD 56, Functionalis, Human Endometrium, Immunocytochemistry, Nerve, Neurofilament

\footnotetext{
${ }^{*}$ Corresponding author.
} 


\section{Introduction}

The presence of nerve fibers in human endometrium is controversial and still remains unsettled [1] [2]. Using fluorescent method for biogenic amines, penetrating nerve fibers in endometrium was identified [1] and a few nerves were present at the endometrium-myometrial interface [2]. Recently, the presence of nerve fibers was implicated on a pain source of endometriosis, in which small nerve fibers were identified throughout basalis and functionalis from women with endometriosis whereas nerve fibers were not observed in functionalis in any women without endometriosis by immunocytochemical staining using protein gene product 9.5 (PGP 9.5) and neurofilament (NF) [3]-[5]. One study with laparotomy-obtained endometrium reported 40\% of women without endometriosis having small numbers by PGP 9.5 immunostaining, which were not positive for NF. Endometrium from women with endometriosis revealed 14 times more small nerve fibers than endometriosis-free women [6]. Thus, the later data suggested the presence of small nerve fibers in the cycling normal endometrium [6] [7]. Using PGP 9.5, another study reported the presence of small nerve fibers in functionalis, basalis and myometrium, which were higher in women hormonally untreated than those hormonally treated women with endometriosis [7]. Thus, more reports support the presence of nerve fibers in normal functionalis in addition to basalis and myometrium [6] [7]. All the previous immunocytochemical studies had been performed with routinely formalin-fixed and paraffin-embedded tissues [2]-[6], which may not detect all nerve fibers whereas frozen section immunocytochemistry revealed consistent presence of lymphatic vessels [8] and venous vessels [9] in endometrium from normally cycling women. The current study aimed to detect the presence of nerve fibers in normally cycling endometrium of different menstrual cycle using NF and CD 56 immunostaining in basalis, functionalis and myometrium. NF is a highly specific immunocythechemical marker for myelinated nerve fibers, which immunostains $\mathrm{A} \sigma, \mathrm{A} \beta, \mathrm{A} \delta$ and $\mathrm{B}$ fibers and $\mathrm{A} \delta$ fibers are small myelinated fibers and transmit sharp, pricking localized pain to CNS [3] [10]. C-fibers are small unmyelinated fibers and transmit dull, aching, burning poorly located pain [3]. CD 56 expression is compatible to N-CAM and is expressed in thin nerve fibers, fine varicose and sensory nerve endings, cell membrane of ganglion cells and young striated muscle cells but thick nerve fibers, perikarya of ganglion cells and adult striated muscle fibers were reportedly CD 56 negative [11].

\section{Materials and Methods}

Uterine tissue was collected from 16 adult women (age range from 37 to 45 years) undergoing elective hysterectomy. Written informed consent was provided by all subjects and ethical approval for tissue collection granted by the Lothian Research Ethics Committee as described before [12]. All women reported regular menstrual cycles (25 - 35 days) and had not received exogenous hormones or used an intrauterine device in the 3 months prior to inclusion in this study. After the uterus had been removed, a wedge of tissue from the inner endometrial surface to the myometrium including the full-thickness of endometrium and contiguous myometrium was taken as described before at an average size of $1 \times 1 \times 0.4 \mathrm{~cm}$ [8] [9] [12]. Fresh wedge tissues were microwave-irradiated for $7 \mathrm{sec}$ in a microwave oven, embedded in OCT, frozen in liquid propane in the liquid nitrogen bath and cryosectioned at 5 - $7 \mu \mathrm{m}$ [12]. Cryosections were mounted on Super Frost Plus slides (Fisher Scientific, Pittsburgh, PA), microwave-irradiated again on ice for $3 \mathrm{sec}$, fixed in $2 \%$ of paraformaldehyde in phosphate buffer at $\mathrm{pH} 7.4$ for 10 to 15 min at room temperature, and immersed twice for 2 min each time in $85 \%$ ethanol [8] [9] [12]. Sections were incubated with blocking serum for $20 \mathrm{~min}$ and then with monoclonal anti-human NF (clone 2F11) or monoclonal CD 56 (Dako, Carpinteria, CA) at 1:100 dilution overnight at $4^{\circ} \mathrm{C}$. After rinsing and immersion in blocking serum again, sections were incubated with second antibody (1:200 dilution) for $30 \mathrm{~min}$ at room temperature. Final visualization was achieved with the alkaline phosphatase Vector blue kit (SK 5300, Vector Laboratories, Burlingame, CA) for blue coloring. Tissue sections were then lightly counterstained with hematoxylin to facilitate identification of cellular components. Since nerve fibers were mostly linear, morphologic analysis was performed for each case for measuring the length of nerve fibers in five randomly selected $10 \times 10=\times 100$ magnified fields in $\mu \mathrm{m}$ using a liner $1 \mathrm{~cm}$ scale with $10 \mu \mathrm{m}$ intervals mounted in the $10 \times$ eye piece as described before [13] for functionalis, basalis and the contiguous myometrium, respectively [13]. The functionalis was divided approximately into upper one-half, a superficial layer, the compacta consisting of densely packed stromal cells around the straight necks of glands, and into a lower one-half layer as the spongiosa, a thick spongy layer containing the tortuous bodies of the glands [14]. The functionalis was studied for the lower functionalis from early-proliferating phase, and for both upper and lower functionalis 
from early-proliferating to late-secretary phase. The total length of nerve fibers was cumulatively calculated, and the mean, SE and $\mathrm{P}$ values with the total numbers of the nerve fibers were calculated for early-proliferative, early-secretary, late-secretary phase and menstrual period (Table 1).

\section{Results}

Generally immunostained nerve fibers were about the same in numbers and sizes by both NF and CD 56 immunostaining and were diffusely and horizontally distributed in the myometrium in all phases (Figures 1-3). There

Table 1. Nerve Fibers in Endometrium and Myometrium.

\begin{tabular}{|c|c|c|c|c|c|c|c|c|c|c|c|c|c|c|c|c|c|c|c|}
\hline & & \multicolumn{12}{|c|}{ Endometrium } & \multirow{2}{*}{\multicolumn{6}{|c|}{ Myometrium }} \\
\hline & & \multicolumn{6}{|c|}{ Functionalis } & \multicolumn{6}{|c|}{ Basalis } & & & & & & \\
\hline & & \multicolumn{3}{|c|}{ NF } & \multicolumn{3}{|c|}{ CD 56} & \multicolumn{3}{|c|}{ NF } & \multicolumn{3}{|c|}{ CD 56} & \multicolumn{3}{|c|}{ NF } & \multicolumn{3}{|c|}{ CD 56} \\
\hline \multicolumn{20}{|c|}{ Day 3 of menstruation } \\
\hline \multirow{2}{*}{\multicolumn{2}{|c|}{ Case }} & & & & & & & \multicolumn{2}{|c|}{ Total } & Mean & \multicolumn{2}{|c|}{ Total } & Mean & \multicolumn{2}{|c|}{ Total } & Mean & Total & \multicolumn{2}{|c|}{ Mean } \\
\hline & & & & & & & & No & Leng & Leng & No & Leng & Leng & No & Leng & Leng & No & Leng & Leng \\
\hline & L & & & & & & & 31 & 1690 & 55 & 32 & 1350 & 42 & 33 & 1480 & 45 & 33 & 2260 & 68 \\
\hline & 2 & & & & & & & 50 & 350 & 67 & 56 & 2430 & 43 & 48 & 2060 & 43 & 60 & 3920 & 65 \\
\hline & 3 & & & & & & & 25 & 850 & 34 & 26 & 990 & 38 & 44 & 2460 & 56 & 49 & 2700 & 55 \\
\hline & ean & & & & & & & 35 & 1963 & 52 & 38 & 1590 & 41 & 42 & 2000 & 48 & 47 & 2960 & 63 \\
\hline & E & & & & & & & 75 & 730 & 9 & 92 & 430 & 3 & 25 & 280 & 3 & 78 & 496 & 4 \\
\hline \multicolumn{20}{|c|}{ Day 5-9 Early Proliferative Phase } \\
\hline & L & & & & & & & 31 & 1690 & 55 & 32 & 1350 & 42 & 33 & 1480 & 45 & 33 & 2260 & 68 \\
\hline & ? & & & & & & & 28 & 2110 & 56 & 18 & 1430 & 79 & 25 & 2160 & 86 & 39 & 3840 & 98 \\
\hline & 3 & & & & & & & 25 & 1280 & 51 & 34 & 1500 & 44 & 34 & 1460 & 41 & 79 & 3580 & 45 \\
\hline & 4 & & & & & & & 15 & 670 & 45 & 23 & 990 & 43 & 23 & 1460 & 63 & 35 & 1690 & 48 \\
\hline & ean & & & & & & & 25 & 1438 & 52 & 27 & 1318 & 52 & 29 & 1625 & 58 & 47 & 2893 & 65 \\
\hline & $E$ & & & & & & & 34 & 307 & 24 & 69 & 113 & 9 & 21 & 18 & 26 & 11 & 517 & 12 \\
\hline \multicolumn{20}{|c|}{ Day 14-22 Early to Mid-Secretary Phase } \\
\hline & $\mathrm{U}$ & 6 & 340 & 37 & 4 & 160 & 40 & & & & & & & & & & & & \\
\hline \multirow[t]{2}{*}{2} & $\mathrm{~L}$ & 13 & 450 & 35 & 12 & 420 & 35 & 34 & 1690 & 50 & 28 & 1920 & 69 & 41 & 2020 & 35 & 33 & 1950 & 59 \\
\hline & $\mathrm{U}$ & 4 & 120 & 30 & 4 & 120 & 30 & & & & & & & & & & & & \\
\hline \multirow[t]{2}{*}{3} & $\mathrm{~L}$ & 9 & 530 & 59 & 62 & 360 & 60 & 49 & 2620 & 53 & 33 & 1900 & 57 & 56 & 2820 & 49 & 42 & 2540 & 61 \\
\hline & $\mathrm{U}$ & 0 & 0 & 0 & 0 & 0 & 0 & & & & & & & & & & & & \\
\hline 4 & $\mathrm{~L}$ & 30 & 1650 & 55 & 31 & 1540 & 50 & 46 & 1730 & 38 & 46 & 1580 & 34 & 56 & 2130 & 59 & 50 & 2030 & 46 \\
\hline & $\mathrm{U}$ & 4 & 120 & 30 & 5 & 220 & 44 & & & & & & & & & & & & \\
\hline & ean & $19^{\mathrm{a}}$ & $900^{\mathrm{a}}$ & $48^{c}$ & $\mathrm{~L}^{3} 2^{\mathrm{b}}$ & $800^{\mathrm{b}}$ & $47^{\mathrm{c}}$ & 45 & 2115 & 7 & 43 & 2207 & 53 & 51 & 2682 & 52 & 46 & 2610 & 55 \\
\hline & $\mathrm{E}$ & 4 & 274 & 6 & L11 & 274 & 5 & 4 & 238 & 7 & 8 & 414 & 7 & 4 & 400 & 7 & 6 & 416 & 5 \\
\hline & Tean & $4^{\mathrm{d}}$ & $69^{d}$ & $24^{\mathrm{f}}$ & $\mathrm{U} 3^{\mathrm{d}}$ & $125^{\mathrm{d}}$ & $29^{f}$ & & & & & & & & & & & & \\
\hline & SE & 2 & 30 & 8 & U 1 & 46 & 10 & & & & & & & & & & & & \\
\hline
\end{tabular}




\begin{tabular}{|c|c|c|c|c|c|c|c|c|c|c|c|c|c|c|c|c|c|c|c|}
\hline \multicolumn{20}{|c|}{ Day 25-26 Late-Secretary Phase } \\
\hline \multirow[t]{2}{*}{1} & $\mathrm{~L}$ & 22 & 360 & 39 & 22 & 920 & 42 & 43 & 1840 & 43 & 34 & 1640 & 47 & 65 & 2300 & 52 & 41 & 2410 & 59 \\
\hline & $\mathrm{U}$ & 10 & 340 & 34 & 4 & 160 & 40 & & & & & & & & & & & & \\
\hline \multirow[t]{2}{*}{2} & $\mathrm{~L}$ & 27 & 1930 & 53 & 27 & 1300 & 48 & 65 & 2980 & 46 & 50 & 2620 & 52 & 74 & 3640 & 49 & 48 & 3050 & 63 \\
\hline & $\mathrm{U}$ & 18 & 540 & 30 & 4 & 120 & 30 & & & & & & & & & & & & \\
\hline \multirow[t]{2}{*}{3} & $\mathrm{~L}$ & 28 & 1160 & 41 & 22 & 860 & 39 & 45 & 2900 & 64 & 41 & 2410 & 59 & 38 & 2270 & 60 & 45 & 2340 & 52 \\
\hline & $\mathrm{U}$ & 13 & 310 & 24 & 10 & 340 & 34 & & & & & & & & & & & & \\
\hline \multirow[t]{2}{*}{4} & $\mathrm{~L}$ & 34 & 1760 & 51 & 24 & 1230 & 31 & 44 & 2130 & 48 & 39 & 1450 & 37 & 45 & 2330 & 52 & 38 & 2410 & 42 \\
\hline & $\mathrm{U}$ & 23 & 840 & 36 & 9 & 330 & 37 & & & & & & & & & & & & \\
\hline \multirow[t]{2}{*}{5} & L & 22 & 1010 & 46 & 23 & 900 & 39 & 43 & 2770 & 64 & 32 & 1790 & 56 & 34 & 1440 & 42 & 36 & 1870 & 52 \\
\hline & $\mathrm{U}$ & 29 & 1250 & 43 & 16 & 700 & 44 & & & & & & & & & & & & \\
\hline \multirow{2}{*}{\multicolumn{2}{|c|}{$\begin{array}{l}\text { LMean } \\
\text { LSEM }\end{array}$}} & $27^{\mathrm{a}}$ & $1336^{\mathrm{a}}$ & $46^{c}$ & $24^{\mathrm{a}}$ & $1042^{\mathrm{a}}$ & $40^{c}$ & 48 & 2524 & 53 & 39 & 1976 & 50 & 51 & 2396 & 51 & 42 & 2416 & 58 \\
\hline & & 2 & 207 & 3 & 9 & 92 & 2 & 4 & 23 & 4 & 3 & 23 & 4 & 8 & 352 & 3 & 2 & 188 & 3 \\
\hline \multicolumn{2}{|c|}{ UMean } & $19^{\mathrm{d}}$ & $656^{\mathrm{e}}$ & $33^{f}$ & $7^{\mathrm{d}}$ & $330^{\mathrm{d}}$ & $37^{f}$ & & & & & & & & & & & & \\
\hline \multicolumn{2}{|c|}{ USEM } & 3 & 176 & 3 & 3 & 100 & 2 & & & & & & & & & & & & \\
\hline
\end{tabular}

U: Upper functionalis, L: Lower functionalis. P values between lower functionalis and basalis: a $<0.005$, b $<0.01$, c: non-significant. P values between upper functionalis and lower functionalis: $\mathrm{d}<0.001$, e $<0.01$, f: non-significant.

were many scattered small nerve fibers in the basalis, located mostly horizontally in the residual thin basalis, adjacent to basalis glands in Day 3 of endometrium by both NF and CD 56 immunostaining, and each nerve fiber measured 30 - $46 \mu \mathrm{m}$, slightly shorter than those in myometrium (Figure 1, Table 1). The smaller nerve fibers in basalis were darker stained by CD 56 than NF immunostaining (Figure 1). The surface of the basalis endometrial gland was also immunostained for CD 56, suggestive of a regenerative process in an early functionalis on the top of basalis, containing a few nerve fibers (Figure 1). There were numerous, small and round CD 56 positive macrophages in the basalis stroma of Day 3 of endometrium (Figure 1). The total numbers and sizes of nerve fibers were about the same in basalis and myometrium (Table 1). Day 5 - 8 endometrium consisted of mainly the basalis with scattered smaller nerve fibers than those in myometrium by less total length and less mean sizes of the nerve fibers (Figure 2, Table 1). The surface of the thin functionalis gland was positive for CD 56 (Figure 2). Day 14 - 22 endometrium showed the distinct presence of functionalis, mostly consisting of lower functionalis and thinner upper functionalis, the latter was negative for CD 56 immunostaining (Figure 2). Basalis showed some small nerve fibers, less than those in myometrium (Figure 2). The lower functionalis contained five times and six times more nerve fibers with about twice the length than those in the upper-functionalis by NF and CD 56 immunostaining, respectively, and were located mostly longitudinal to the uterine cavity (Figure 2, Table 1). Day 25 - 26 of endometrium showed ever increasing length of functionalis, consisting of about one half each of the upper-and lower-functionalis, respectively (Figure 3). The lower one-half functionalis contained many scattered, longitudinal nerve fibers, consisting of much more scattered nerve fibers at about twice by NF and more than three times by CD 56 immunostaining of those in the upper functionalis (Table 1). The less numerous nerve fibers in upper functionalis were about $60 \%$ those in the lower functionalis and about $40 \%$ those of the basalis, mostly located adjacent to the functionalis glands (Figure 3, Table 1). All functionalis glands were negative for CD 56 immunostaining (Figure 3). Myometrium was completely negative for NF and CD 56 immunostaining (Figures 1-3). Nerve fibers were mostly non-myelinated nerve fibers mixed with occasional myelinated nerve fibers, as both the peroxidase and alkaline phosphatase method consistently immunosatained myelinated nerve fibers but non-myelinated nerve fibers were positively immunostained by only alkaline phosphatase method using NF as a marker (Figure 4).

\section{Discussion}

The presence of nerve fibers in human endometrium has been previously focused on the pathogenesis of endometriosis-associated pelvic pain [3]-[6], however, the relationship between pelvic pain and endometriosis re- 

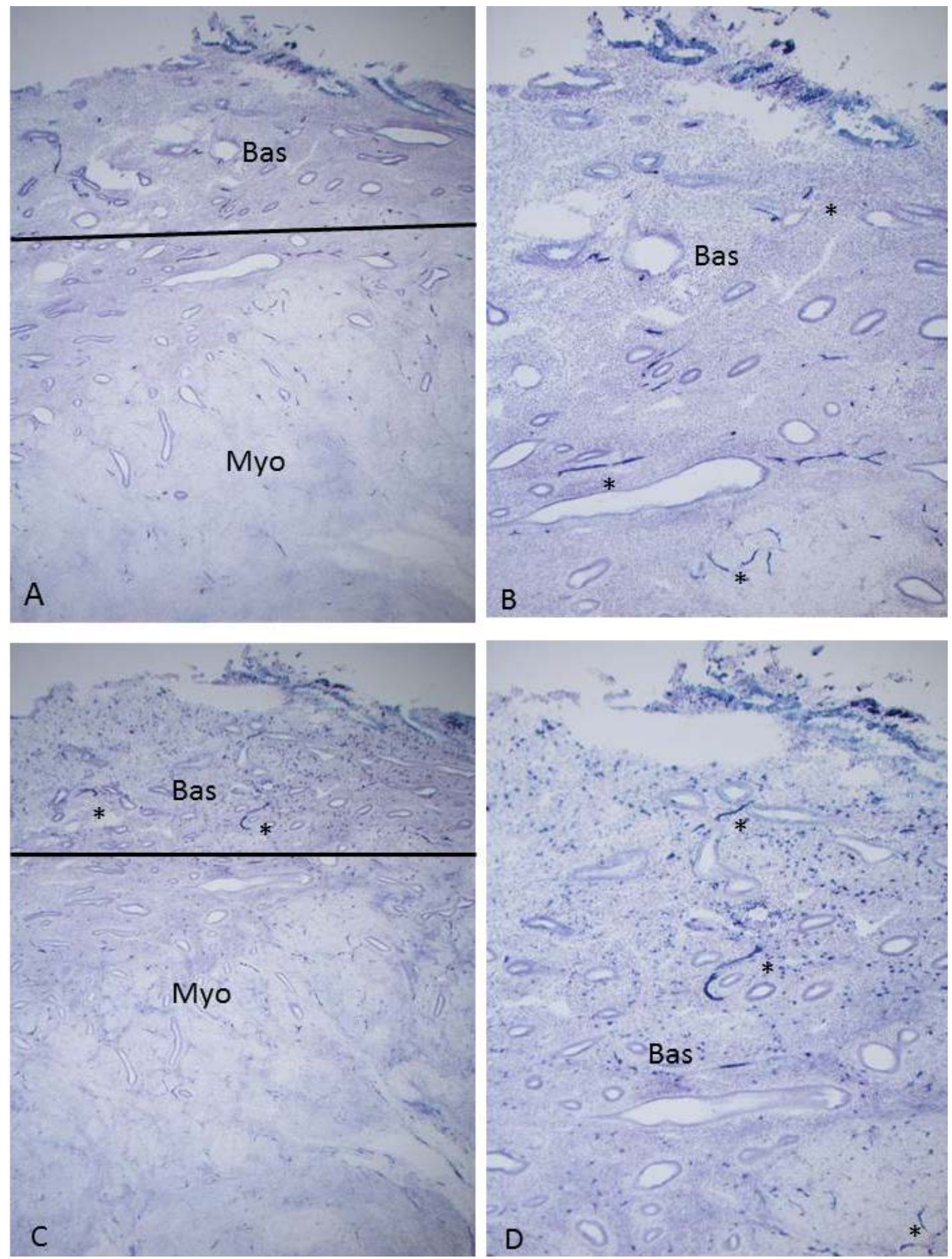

Figure 1. Day 3 after the onset of menstruation, Case 3. There were diffusely, mostly horizontally scattered, numerous small and large nerve fibers in myometrium (Myo) and there were also relatively less, but diffusely distributed small and large nerve fibers in basalis (Bas) by both NF and CD 56 immunostaining. CD 56 immunostining also showed abundantly numerous macrophages in basalis. (A) and (C) $\times 50$; (B) and (D) $\times 100$; (A) and (C) NF; (B) and (D) CD 56 immunostaining. 

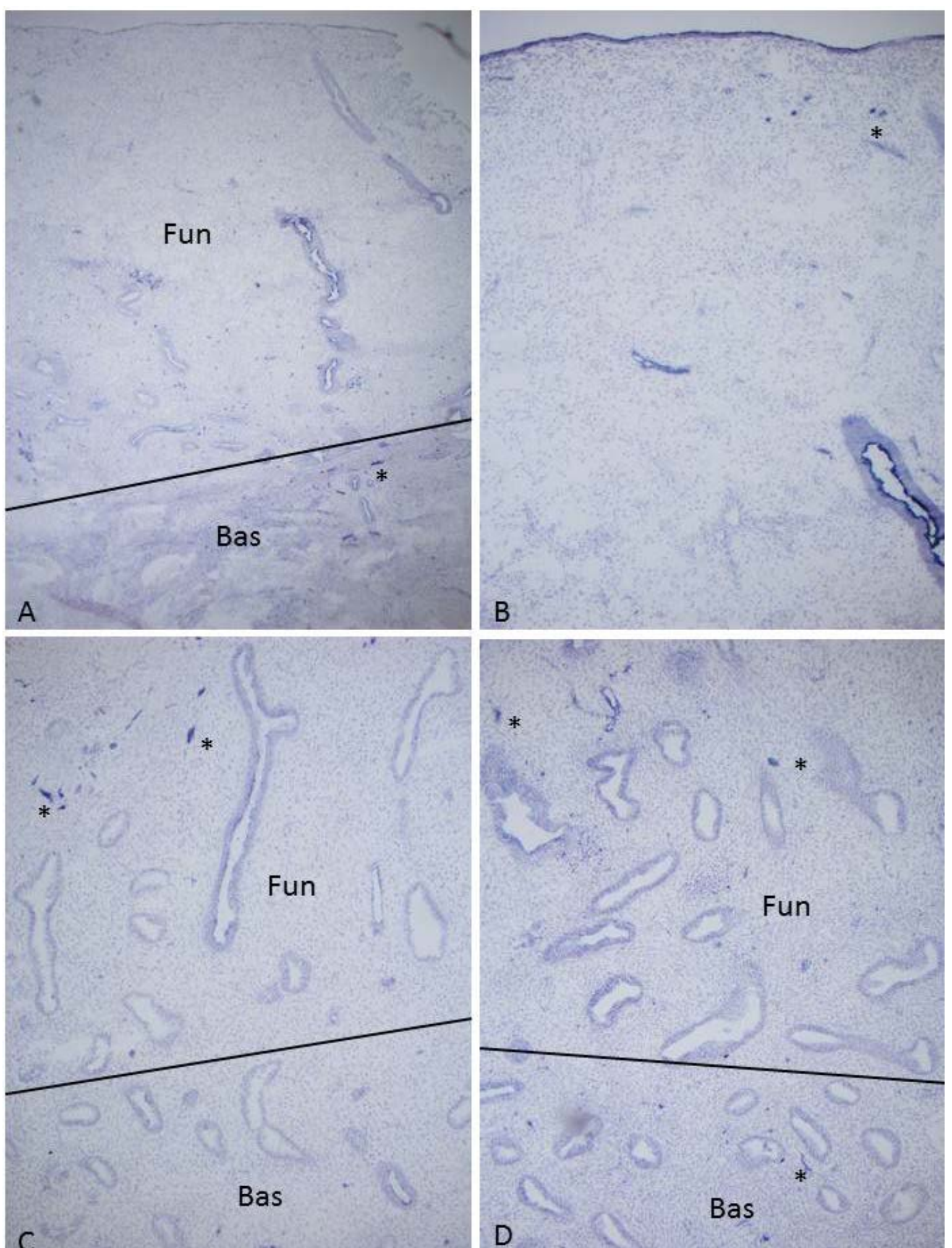

Figure 2. Early proliferative phase, Day 9, Case 4, (A) and (B) and mid-proliferative phase, Day 14, Case 1, (C) and (D) Day 9 of endometrium showed thin functionalis, which contained no nerve fibers ( ${ }^{*}$ ) by both NF and CD 56 immunostaining, the latter immunostaining was positive for the surface of the functionalis glands. Day 14 functionalis showed a few, longitudinally scattered small nerve fibers by both NF and CD 56 immunostaining ( ${ }^{*}$ ). (A) and (C) NF, (B) and (D) CD 56 immunostaining. 

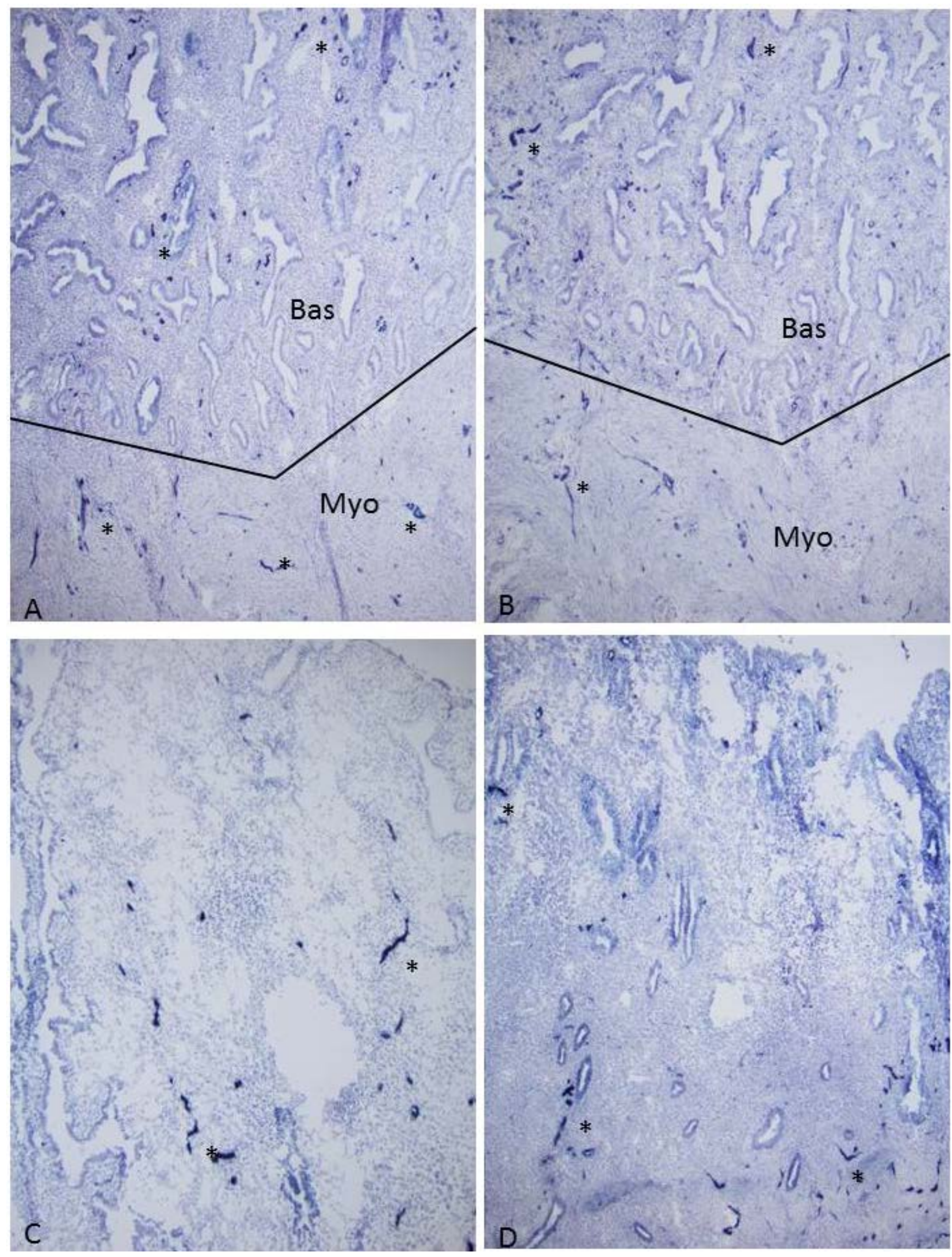

Figure 3. Late-secretary phase, Day 26, Case 2 Myometrium (Myo) and basalis (Bas) showed diffuse horizontal, numerous small and large nerve fibers ( $\left(^{*}\right)$ by both NF and CD 56 immunostaining. Functionalis showed many, longitudinal nerve fibers $\left(^{*}\right)$ in the lower functionalis compared with less but definitely present nerve fibers in the upper functionalis. (C) and (D) Upper functionalis; (A) and (C) NF, B and D: CD 56 immunostaining. 

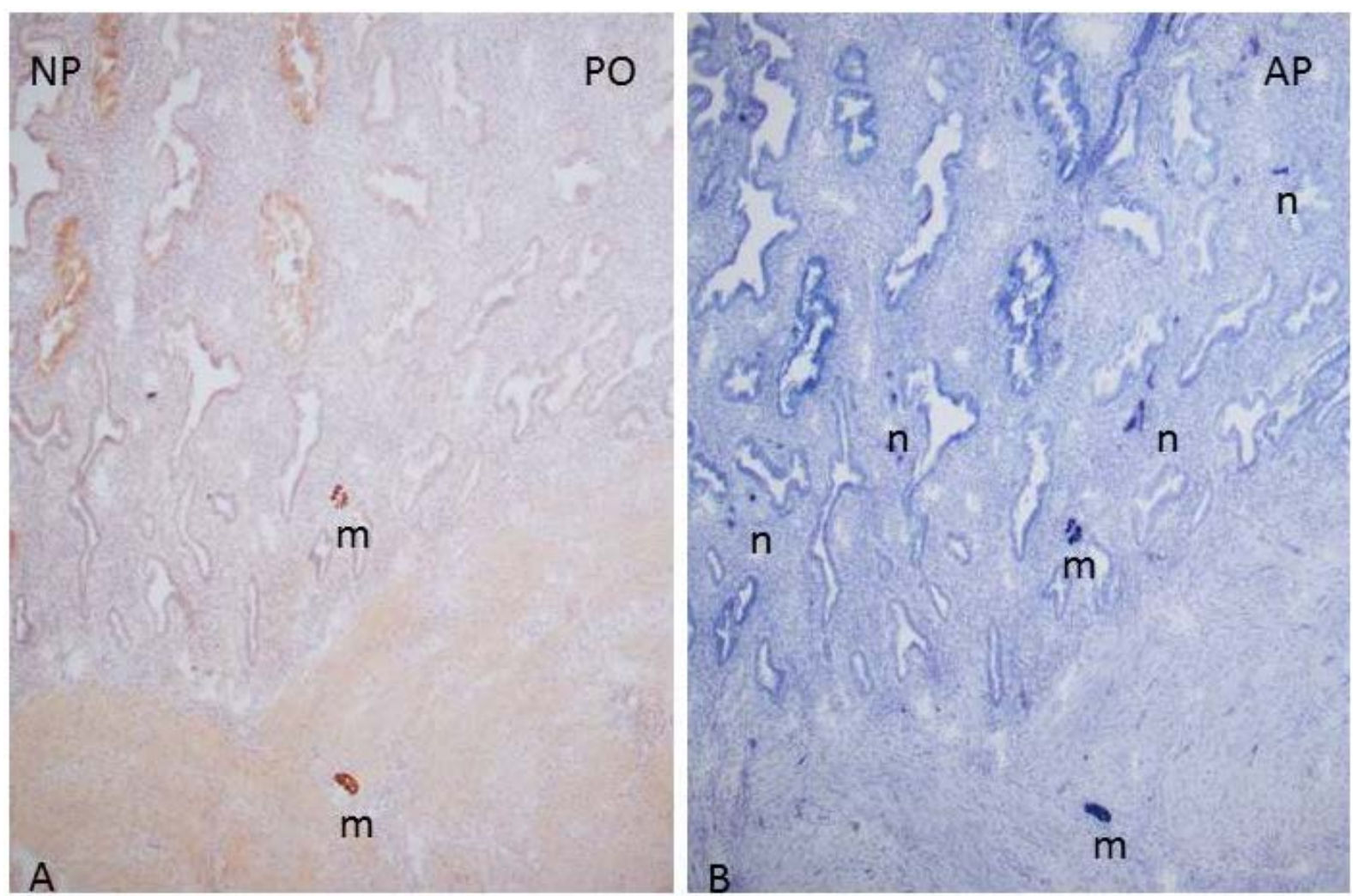

Figure 4. Late-secretary phase, Day 25, Case 1 myelinated nerves $(\mathrm{m})$ were immunostrained for NF using both peroxidase method (A) and alkaline phosphatase method (B), however, unmyelinated nerve fibers were immunostained only by alkaline phosphatase method not by peroxide method. (A) NF by peroxidase method; (B) NF by alkaline phosphatase method.

mains unclear [4]. Pelvic pain is the most common and specific symptom for endometriosis, which occurs about $10 \%$ of women and endometriosis is a major cause of chronic pelvic pain [3]-[6]. Endometriosis-associated pain may be caused by peritoneal inflammation, adhesion and specific innervation of endometriotic lesions and is correlated with the presence of deep infiltrating disease [15] [16]. However, there is no clear correlation between pelvic pain and degree of endometriosis [17]. Endometriosis is an estrogen-dependent disease, and current medical treatments suppress estrogen synthesis and medications such as Damazol, GnRH analogue, combined oral contraceptives and progesterone have been used to treat endometriosis-associated pelvic pain [18]. The classic visceral pain felt deep within abdominal or pelvic cavities is characteristically diffused or dull and poorly localized, and the cause of this pain is not clear [16]. Several investigators reported the presence of nerve fibers in endometriotic lesions [3]-[6] [16]-[20]. The presence of nerve fibers in eutopic human endometrium has been controversial: Nerve fibers have been detected in endometrium from women with endometriosis using PGP 9.5 and NF as markers, suggesting a role of endometrial nerve fibers for pathophysiology of endometriosis-associated pelvic pain [3]-[6] [14]. Tamburro et al. reported nerve fibers expressing transforming growth factor $\beta 1$ [TGF- $\beta 1$ ] in endometriotic lesion from women with dysmenorrhea associated with endometriosis [16]. Tulandi et al. reported NF-immunopositive nerve fibers in endometriotic lesions, in which the distance between endometrial glands and nerve fibers was closer in women with pain than women with no pain [20]. Regarding to the eutopic endometrial nerves, one study reported $40 \%$ of women without endometriosis having small numbers of PGP 9.5 positive nerve fibers [6] and another study reported the density of small nerve fibers being 14 times higher in women with endometriosis compared with control endometriosis-free women using laparotomy-obtained endometrial tissues [7]. Thus, the eutopic endometrium from women with endometriosis is biologically different from women with a normal pelvis [19]-[22]. The presence of nerve fibers in the eutopic endometrium is used for diagnosing pelvic pain-associated endometriosis by some authors [19]-[22]. In deep infiltrating endometriosis which endometriotic lesions penetrate into the retroperitoneal space of the wall of pelvic organs for a distance of $5 \mathrm{~mm}$ or more, four times more nerve fibers were detected than in peritoneal endometriosis [20]. 
The association between pelvic pain and deep endometriosis is much stronger than in other types of endometriosis [19] [20]. The eutopic endometrium from women with endometriosis is innervated by sensory C, a mixture of sensory $\mathrm{C}$ and sensory $\delta$, and adrenergic fibers in basalis whereas myometrium is innervated by a mixture of sensory $\mathrm{A} \delta$, sensory C, adrenergic and cholinergic fibers [20]. All previously reported studies had been performed with the routinely formalin-fixed and paraffin-embedded tissues [3]-[7]. We aimed to study to identify nerve fibers with cryosections, which were used previously for detecting lymphatic vessels and venous vessels obtaining better results than using the routinely formalin-fixed paraffin-embedded tissue sections [8]. Using routinely formalin-fixed and paraffin-embedded tissues, Boker et al. reported $90 \%$ of uteri with endometriosis showing more nerve fibers, which were not homogeneously distributed and the degree of immunostaining was the best with rabbit PGP 9.5, followed by rabbit NPY, monoclonal CGRP, rabbit SP and rabbit VIP but no immunostaining by monoclonal NF using alkaline phosphatase for detecting method [6], as we used in this study. Our immunostaining with cryosections produced more homogenous and diffusely distributed nerve fibers in functionalis, basalis and myometrium (Figures 1-3), which proved to be a better and superior staining than the routinely paraffin-embedded tissues for nerve fibers, lymphatic vessels and venous vessels [8] [9]. Both Boker et $a l$. and Tokushige et al. used neural markers for sensory C, A $\delta$, adrenergic and cholinergic nerve fibers in the functionalis with several nerve markers including PGP 9.5, NF, substance P, vasointestinal polypeptide, neuropeptide Y and calcitonin gene-related peptide [6] [22] [23]. The majority of endometrial nerve fibers were non-myelinated small nerves fibers, some of which may correspond to small sensory nerve fibers for transmitting pain (Figure 4). Tokushige et al. studied the distribution of nerve fibers in functionalis, basalis and myometrium with the routinely paraffin-embedded full-thickness uterine blocks using rabbit PGP 9.5, monoclonal NF, rabbit SP, rabbit VIP and rabbit NPY: In the uterine tissues from hormonally treated women with endometriosis, nerve fibers density was $0.2 \pm 0.7 \mathrm{~mm}^{2}$ for functionalis, $0.9 \pm 1.3 \mathrm{~mm}^{2}$ for basalis and $1.5 \pm 0.8 \mathrm{~mm}^{2}$ for myometrium, respectively, which were much less than the hormonally untreated uterine tissues from endometriosis women: $11 \pm 5 \mathrm{~mm}^{2}$ for functionalis, $18 \pm 8 \mathrm{~mm}^{2}$ for basalis and $3 \pm 1 \mathrm{~mm}^{2}$ for myometrium [7]. These data showed markedly reduced nerve fibers in hormonally treated uterine tissues compared with the hormonally untreated uterine tissues, particularly a marked reduction of nerve fibers by the hormone treatment to $0.2 \pm 0.7$ $\mathrm{mm}^{2}$ from $11 \pm 5 \mathrm{~mm}^{2}$ in the functionalis [7]. Their nerve fiber density in functionalis, basalis and myometrium from hormonally treated uterine tissues presented large variation with large SE values but roughly paralleled with the total nerve fiber length in normal endometrium from women without endometriosis presented in this current study, showing most nerve fibers in myometrium followed by in basalis and in functionalis of the late-secretary phase (Table 1). As mentioned above, many investigators had used rabbit PGP 9.5 for immunostaining nerve fibers with the routinely paraffin-embedded tissues, which also immunosatained nerve fibers and neuroendocrine tumors [24] but this polyclonal antibody did not immunostain nerve fibers with cryosections at our hand, thus we used the alternative markers, including NF and CD 56 in this study. As shown in this study, the majority of small nerve fibers were unmyelinated nerves with a few scattered myelinated nerves observed in endometrium (Figure 4). Myelinated nerves were readily localized by NF immunostaining using both peroxidase and alkaline phosphatase detecting method but unmyelinated nerve fibers were consistently immunostained only by alkaline phosphatase detecting method (Figure 4). With cryosections, our results showed the consistent presence of horizontal nerve fibers in myometrium and basalis, which appeared to be non-cycling nerve fibers (Table 1). In functionalis, there were few nerve fibers in the very thin functionalis from Day 5 - 8 proliferative phase (Figure 1(C) and Figure 1(D)), but in 14 - 22 Day of endometrium, there were about one-half of longitudinally growing nerve fibers in the upper one-half functionalis than those in the lower one-half functionalis (Table 1). In late-secretary phase, Day 25 to 26 of endometrium, there were more longitudinal small nerve fibers in the upper one-half functionalis, which were still less than those of lower one-half functionalis, the latter appeared to be growing from basalis. Comparing the nerve fibers between in functionalis and in myometrium, myometrium consistently contained three times more horizontal nerve fibers than in the lower one-half of functionalis (Table 1). As seen in this study, innervation in the endometrium is significantly less than in the myometrium, and nerve fibers in the endometrium are mainly associated with blood vessels located at the myometrium-endometrial border [7]. Neural modulation in the uterus is related to sex steroid concentrations, and a reduction in nerve fibers is observed when estrogen levels are high during ovulatory cycle and is restored during menstruation in a rat study [25]. Uterine innervation is mainly associated with blood vessels, but many nerve fibers lie free within myometrium and in endometrial stroma [26] as seen in this study as well (Figures 1-3). The parent intramyometrial nerve fibers are unaffected during menstrual cycle [26] [27]. Small 
nerve fibers in the uterus are not only autonomic nerves but also sensory nerves, and the latter travels through the vascular zone to innervate smooth muscle and endometrium, and transmit pelvic pain [28]. Among the total nerve population visualized by PGP 9.5 immunostaining, only the sympathetic nerves account for the actual decrease of nerve fibers not affecting sensory nerves during the ovulatory phase in a rat study [28]. What can we explain this different nerve fiber distribution between in endometrium and in myometrium? Nerve fibers in myometrium are non-cycling nerve fibers by consistent same numbers and lengths throughout menstrual cycle as shown in Table 1 whereas nerve fibers in functionalis are cycling, which fluctuate according to the menstrual cycle: Nerve fibers were absent in early proliferative phase and increased from early to late secretary phase in the concomitantly growing functionalis (Table 1). Since blood vessels and nerve fibers develop in coordinated patterns, cyclic changes of endometrial arteries of the human endometrium are well documented, with which nerve fibers develop and regress in coordinated patters [29]: A gradual increase in arborization of coiling of spiral arteries during proliferation in ovulatory period and the spiral growths parallel the gradual increase in length and coiling of endometrial glands in post-ovulatory period [30]. Nerve fibers likely parallel the growth of arteries but do not grow as fast as arteries especially in functionalis due to a slower growth rate of nerve fiber, since growth of endometrial glands and arteries is faster by estrogen effects than relatively slower growing nerve fibers [13]. The biological significance of endometrial innervation may play important roles in pathophysiology through sensory nervous system for pelvic pain during menstruation and endometriosis as well as for infertility and spontaneous abortion [2].

\section{Acknowledgements}

I want to express our sincere thanks to Dr. Hilary O.D. Critchley, University of Edinburgh, UK for providing the human uterine tissues and Drs. Robert Brenner and O.V. Slayden for allowing us to use their research laboratory at National Oregon Primate Center, Beaverton, OR. This study was supported in part by ONPRC Core grant: NIH 000163.

\section{References}

[1] Dallenback, F.D. and Vonderlin, D. (1973) The Innervation of the Human Endometrium. Archiv für Gynäkologie, 215, 365-376.

[2] Latini, C, Frontini, M., Morroni, M., et al. (1998) Remodeling of Uterine Innervation. Cell and Tissue Research, 334, 1-6. http://dx.doi.org/10.1007/s00441-008-0657-X

[3] Tokushige, N., Markham, R., Russels, P. and Fraser, I.S. (2006) High Density of Small Nerve Fibres in the Functional Layer of the Endometrium in Women with Endometriosis. Human Reproduction, 21, 782-787. http://dx.doi.org/10.1093/humrep/dei368

[4] Tokushige, N., Al-Jefout, M., Salih, H. and Fraser, I.S. (2007) Endometrial Nerve Fibres in Endometriosis. Iranian Journal of Reproductive Medicine, 5, 81-88.

[5] Tokushige, N., Markham, R., Russel, P. and Fraser, I.S. (2007) Different Types of Small Nerve Fibers in Eutopic Endometrium and Myometrium in Women with Endometriosis. Fertility and Sterility, 88, 795-803. http://dx.doi.org/10.1016/j.fertnstert.2006.12.078

[6] Boker, A., Kyama, C.M., Vercruysse, L., et al. (2009) Density of Small Diameter Sensory Nerve Fibers in Endometrium: A Semi-Invasive Diagnostic Test for Minimal to Mild Endometriosis. Human Reproduction, 24, 3025-3032.

[7] Tokushige, N., Markham, R., Russel, P., et al. (2008) Effects of Hormonal Treatment in Nerve Fibers in Endometrium and Myometrium in Women with Endometriosis. Fertility and Sterility, 90, 1589-1598. http://dx.doi.org/10.1016/j.fertnstert.2007.08.074

[8] Tomita, T. and Mah, K. (2014) Cyclic Changes of Lymphatic and Venous Vessels in Human Endometrium. Open Journal of Pathology, 4, 5-12. http://dx.doi.org/10.4236/ojpathology.2014.41002

[9] Tomita, T. and Mah, K. (in review) Cyclic Change of Lymphatic and Venous Vessels in Human Endometrium.

[10] Schlaeffer, W.W. (1987) Neurofilaments: Structure, Metabolism and Implications in Disease. Journal of Neuropathology \& Experimental Neurology, 46, 117-129. http://dx.doi.org/10.1097/00005072-198703000-00001

[11] Mechtersheimer, G., Stauder, M. and Moller, P. (1991) Expression of the Natural Killer Cell-Associated Antigens CD56 and CD57 in Human Neural and Striated Muscle Cells and Their Tumors. Cancer Research, 51, 1300-1307.

[12] Brenner, R.M., Slayden, O.D., Rogers, W.H., et al. (2003) Immunocytochemical Assessment of Mitotic Activity with an Antibody to Phosphorylated Histone H3 in the Macaque and Human Endometrium. Human Reproduction, 18, 1185- 
1193. http://dx.doi.org/10.1093/humrep/deg255

[13] Tomita, T. (2012) Islet Amyloid Polypeptide in Pancreatic Islets from Type 2 Diabetic Subjects. Islets, 4, 223-232. http://dx.doi.org/10.4161/isl.20477

[14] Brenner, R.M. and Slayden, O.D. (1994) Cyclic Changes in Primate Oviduct and Endometrium. In: Knobeil, K. and Neil, J.D., Eds., The Physiology of Reproduction, Raven Press, New York, 541-568.

[15] Anaf, V., Chapron, C., El Nakadi, E., et al. (2006) Pain, Mast Cells and Nerves in Peritonea, Ovarian and Deep Infiltrating Endometriosis. Fertility and Sterility, 86, 1336-1343. http://dx.doi.org/10.1016/j.fertnstert.2006.03.057

[16] Merchsner, S., Schwarz, J., Thode, J., et al. (2007) Growth-Associated Protein 43-Positive Sensory Nerve Fibers Accompanied by Immature Vessels Are Located in or near Peritoneal Endometriotic Lesions. Fertility and Sterility, 88, 581-589. http://dx.doi.org/10.1016/j.fertnstert.2006.12.087

[17] Chapron, C., Faucoier, A., Dubuisson, J.B., et al. (2003) Deep Infiltrating Endometriosis: Relation between Severity of Dysmenorrhea and Extent of Disease. Human Reproduction, 18, 760-766. http://dx.doi.org/10.1093/humrep/deg152

[18] Amserdam, L.L., Gentry, W., Wolf, M., et al. (2005) Anastrazole and Oral Contraceptives: A Novel Treatment for Endometriosis. Fertility and Sterility, 84, 300-304. http://dx.doi.org/10.1016/j.fertnstert.2005.02.018

[19] Talundi, T., Felemban, A. and Chen, M.F. (2001) Nerve Fibers and Histopathology of Endometriosis-Harboring Peritoneum. Journal of the American Association of Gynecologic Laparoscopists, 113, 105-107.

[20] Wang, G., Tokushige, N., Markham, R. and Fraser, I.S. (2009) Rich Innervation of Deep Infiltrating Endometriosis. Human Reproduction, 24, 827-834. http://dx.doi.org/10.1093/humrep/den464

[21] Tokushige, N., Markham, R., Russell, P. and Fraser, I.S. (2006) Nerve Fibers in Peritoneal Endometriosis. Human Reproduction, 21, 3001-3007. http://dx.doi.org/10.1093/humrep/del260

[22] Tokushige, N., Markham, R., Russell, P. and Fraser, I.S. (2007) Different Types of Nerve Fibers in Eutopic Endometrium and Myometrium in Women with Endometriosis. Fertility and Sterility, 88, 795-803. http://dx.doi.org/10.1016/j.fertnstert.2006.12.078

[23] Tran, L.V., Tokushige, N., Berbic, M., Markham, R. and Fraser, I.S. (2009) Macrophages and Nerve Fibers in Peritoneal Endometriosis. Human Reproduction, 24, 835-841. http://dx.doi.org/10.1093/humrep/den483

[24] Tomita, T. (2013) PGP 9.5 Immunocytochemical Staining for Pancreatic Endocrine Tumors. Islets, 5, 122-128. http://dx.doi.org/10.4161/isl.25351

[25] Zoubina, E.V., Fan, Q. and Smith, P.G. (1998) Variations in Uterine Innervation during the Estrous Cycle in Rat. Journal of Comparative Neurology, 197, 561-571.

[26] Haase, E.B., Buchman, J., Tiez, A.E. and Schramm, L.P. (1997) Pregnancy-Induced Uterine Neuronal Degeneration in the Rat. Cell and Tissue Research, 288, 293-306. http://dx.doi.org/10.1007/s004410050815

[27] Zoubina, E.V. and Smith, P.G. (2000) Axonal Degeneration and Regeneration in Rat Uterus during the Estrous Cycle. Autonomic Neuroscience: Basic and Clinical, 84, 176-185. http://dx.doi.org/10.1016/S1566-0702(00)00209-5

[28] Arese, M., Serini, G. and Bussolino, F. (2011) Nervous Vascular Parallels: Axons Guidance and Beyond. International Journal of Developmental Biology, 55, 439-445. http://dx.doi.org/10.1387/ijdb.103242ma

[29] Buga, G.A.B. (2007) The Normal Menstrual Cycling. In: Kruger, T.F. and Batha, M.H., Eds., Clinical Gynecology, 3rd Edition, Jata Co., Cape Town, 73-87.

[30] Mutter, G.L. and Frenzy, A. (2002) Anatomy and Histology of the Uterine Corpus. In: Kurlman, R.J., Ed., Blaunstein's Pathology of the Female Genital Tract, 5th Edition, Springer Verlag, New York, 383-419. 